\title{
PROCESOS PSICOLÓGICOS DE LA LECTURA EN ESTUDIANTES DE PRIMARIA DEL CALLAO, SEGÚN NIVEL SOCIOECONÓMICO Y GÉNERO
}

\author{
PSYCHOLOGICAL PROCESSES OF READING IN ELEMENTARY STUDENTS OF \\ THE CALLAO ACCORDING TO SOCIOECONOMIC STATUS AND GENDER
}

Esther Velarde C. ${ }^{1}$, Ricardo Canales G. , Magali Meléndez J. y Susana Lingán H.

Universidad Nacional Mayor de San Marcos, Perú

(RECIBIDO 2/10/2012, ACEPTADO 25/04/2013)

\begin{abstract}
RESUMEN
El objetivo de la presente investigación fue conocer si existen diferencias en los procesos psicológicos de la lectura según el nivel socioeconómico y género en 965 estudiantes de $1^{\circ}$ a $6^{\circ}$ grado de primaria de escuelas particulares y estatales del Callao. Para dicho fin se adaptó y crearon baremos para la batería de evaluación de los procesos lectores-revisada (PROLEC-R) de Cuetos, et al. (2007). Se empleó un diseño descriptivo comparativo. Se encontraron diferencias significativas según nivel socioeconómico en todos los grados en comprensión de textos y comprensión oral, además en identificación de letras y comprensión de oraciones (excepto en el $6^{\circ}$ ) y en signos de puntuación (excepto en segundo grado). No se hallaron diferencias en la tarea de identificar palabras "igual-diferente" y lectura de palabras (excepto en $1^{\circ}$ ) y en lectura de pseudopalabras (excepto $1^{\circ}$ y $3^{\circ}$ ) y estructuras gramaticales (excepto $1^{\circ}$ y $2^{\circ}$ ). En cuanto al género, se halló diferencias en comprensión oral a favor de los varones. Se elaboraron puntuaciones normativas encontrándose un rendimiento más bajo en todos los procesos psicológicos de la lectura en el nivel socioeconómico E.
\end{abstract}

Palabras clave: procesos psicológicos de la lectura, estrategia visual, estrategia fonológica, nivel socioeconómico

\begin{abstract}
The objective of this research was to know if there were differences in the psychological processes of reading regarding the socio-economic level and gender of 965 students from $1^{\text {st }}$ to $6^{\text {th }}$ grade of primary from private and public schools of Callao. For this purpose we adapted and created scales for the battery for Reading processes evaluation - revised (PROLEC-R) by. Cuetos, et al (2007). A comparative descriptive design was used. Significant differences according the socioeconomic level in all grades were found in reading comprehension and oral comprehension. It was also found differences in identification of letters and understanding of sentences (except in $6^{\text {th}}$ ) and punctuation (except in second grade). There are no diffe-
\end{abstract}

1 Docente asociada de la Facultad de Educación de la UNMSM. Email: emvelarde@hotmail.com 
rences in the task of identifying "same-different" words and reading of words (except for $1^{\text {st }}$ grade) and pseudowords (except in $1^{\text {st }}$ and $3{ }^{\text {rd }}$ ) reading and grammatical structures (except $1^{\text {st }}$ and $\left.2^{\text {nd }}\right)$. Regarding the gender, only difference found was in listening comprehension in favouring the boys. Normative scores were produced lower performance found in all the psychological processes of the reading in the socio-economic E level.

Keywords: reading psychological processes, visual reading strategy, phonemic reading strategy, level socioeconomic

\section{INTRODUCCIÓN}

El nivel de dominio que presentan nuestros estudiantes sobre la lectura constituye un grave problema nacional. La última evaluación del Programme for International Student Assessment (OCDE, 2009) exhibe el estado del Perú frente al desempeño de otros países de América Latina. Se observa que en el caso de México, Argentina y Chile sus estudiantes quedaron muy parejos en las pruebas de Comprensión Lectora, Matemáticas y Ciencias. Brasil se situó un poco rezagado mientras que Perú se ubicó en el último lugar. Una de las causas por las cuales no se remontan los resultados nacionales e internacionales es porque el Diseño Curricular Nacional (Ministerio de Educación del Perú, 2009) presenta un modelo de lectura que no se ajusta a los avances científicos de la psicología cognitiva y las neurociencias. Desde el año 1980 ha generalizado en los países del mundo, la implementación de propuestas educativas sobre la base del modelo cognitivo y psicolingüístico de la lectura. Esta se basa en investigaciones que han demostrado la existencia de procesos y operaciones cognitivas que cumplen funciones específicas y se interrelacionan de manera dialéctica y coordinada con el resto, para permitir el logro de una competencia tan compleja como es la de la lectura. Hoy se sabe que existen módulos especializados para el procesamiento fonológico, para el almacenamiento de los grafemas, para la representación léxica-ortográfica, etc. Ello permite entender que si bien la lectura es un proceso que actúa como un todo, presenta funciones específicas que se deben activar adecuadamente para que este procedimiento sea óptimo.

El modelo cognitivo y psicolingüístico de la lectura permite conocer el funcionamiento de cada uno de los procesos psicológicos que intervienen en la lectura: identificación de letras (conocimiento del nombre o sonido de la letra), procesos léxicos o de reconocimiento de palabras o pseudopalabras (dominio de la ruta léxica y fonológica), procesos sintácticos (conocimiento de la estructura gramatical y de los signos de puntuación), procesos semánticos de la lectura (comprensión de oraciones, comprensión de textos y comprensión oral). A partir de este conocimiento es posible identificar cuál o cuáles son los procesos psicológicos de la lectura se encuentran deficientes y, de esta manera, estar en condiciones de plantear pautas para su recuperación.

En la actualidad, el Ministerio de Educación viene realizando estudios censales con alumnos de segundo grado a los cuales diagnostica sobre la base de tres categorías: nivel suficiente (2), nivel previo (1) y antes del previo (menor de 1). Sin embargo 
no informa sobre cuál es el proceso de la lectura que se encuentra deficiente de tal manera que los programas de capacitación docente resultan muy generales y no apuntan a resolver los problemas específicos que serán distintos según las características poblacionales, económicas y socioculturales de los educandos. Por lo tanto es fundamental elaborar un perfil del desempeño en lectura que obtienen los estudiantes y la detección de las dificultades encontradas en cada uno de los procesos psicológicos. Solo así se estará en condiciones de sugerir la aplicación de adecuados programas de intervención que permitirá rehabilitarlos, logrando superar sus dificultades.

Para realizar una medición basada en el enfoque cognitivo y psicolingüístico se requiere de instrumentos que se sustenten en sólidos estudios e investigaciones (Dioses, Evangelista, Basurto, Morales, Alcántara, 2010). Uno de esos instrumentos es la Batería de Evaluación de los Procesos Lectores, Revisada (PROLEC-R) de F. Cuetos et al. (op. cit. 2007). Esta prueba está compuesta por una serie de subtests: identificación de letras (que mide el conocimiento del nombre o sonido de la letra), procesos léxicos o de reconocimiento de palabras o pseudopalabras (que evalúa el dominio de la ruta léxica y fonológica), procesos sintácticos (que informa sobre el conocimiento de la estructura gramatical y la identificación de los signos de puntuación), procesos semánticos de la lectura (que evalúa la comprensión de oraciones, comprensión de textos y comprensión oral). El subtest de identificación de letras estaría comprendido dentro de los denominados procesos de bajo nivel, mientras que la de los procesos léxicos se ubican dentro del nivel medio. Los procesamientos sintáctico y semántico estarían comprendidos dentro de las operaciones de alto nivel que son los responsables de la construcción del significado global del texto (Vega, Carreiras, Gutiérrez-Clavo y Alonso, 1990; Cuetos, 1991; Vega y Cuetos, 1998; Morais, 1998, citados por García \& González, 2000). Cada uno de ellos contiene una serie de operaciones cognitivas, las cuales pueden ser evaluadas para comprobar su estado de funcionamiento, de tal manera que se puedan plantear las medidas efectivas de intervención en cada uno de ellos. Sin embargo, este instrumento no contaba con normas adaptadas a nuestra realidad.

Por otro lado, respecto a la evaluación de los procesos lectores, hay que tomar en cuenta que en el Perú existen factores disortogénicos que perturban el desarrollo normal y óptimo del niño como la pobreza, marginalidad, desnutrición, presencia de bilingüismo sustractivo, carencia de estimulación temprana y la inequidad educativa (González, 1993, 1995c). Estos factores marcan las diferencias en los desempeños en diversas funciones cognitivas y psicolingüísticas entre los niños pertenecientes a nivel socio económico bajo y los de nivel alto. En última evaluación censal 2011 (Ministerio de Educación, ECE 2011) el 50,3\% de los estudiantes de instituciones no estatales alcanzan el Nivel 2 (lo esperado para su grado) frente a un 23,0\% provenientes de instituciones estatales. En el año 2010 fue un 48,6\% frente a un $22,8 \%$ habiéndose obtenido solo un $0,2 \%$ de mejora entre el 2010 y el 2011 en el caso de los alumnos de colegios estatales y un $1,7 \%$ en lo que respecta a los colegios particulares. Las cifras son más alarmantes si comparamos zonas urbanas y rurales. Así tenemos que en el 2011 el 36,3\% de los estudiantes provenientes de colegios localizados en la parte urbana del país alcanzaron el Nivel 2 frente a un 5,8\% que procedían de zonas rurales. (ECE, 2011). 
Complementariamente, la misma Evaluación Censal de Estudiantes, revela diferencias en comprensión lectora según género. Así tenemos que en el ECE 2010 fueron un $26,8 \%$ de varones que alcanzaron el nivel 2 frente a un 30,7\% de mujeres y en el ECE 2011 los varones obtuvieron un 27,2\% de logro en el nivel 2 frente a un $32,4 \%$ en el caso de las mujeres.

En tal sentido en el presente estudio, nos propusimos como objetivo principal, evaluar el funcionamiento de las operaciones y procesos implicados en la lectura y conocer cómo influye la variable socioeconómica y género en la conformación y funcionamiento de cada uno de los procesos lectores.

Para ello se seleccionó un instrumento de evaluación de la lectura de orientación cognitiva y psicolingüística: PROLEC-R, a partir del cual, luego de realizar las adaptaciones requeridas, se han elaborado los baremos respectivos para aplicarlo a nuestra población de estudio.

Adicionalmente a ello, tratamos también de observar la velocidad de la lectura que está estrechamente vinculada con el nivel de dominio alcanzado en la comprensión lectora (Wimmer 1993).

A partir de lo señalado se planteó como hipótesis, las siguientes:

\section{Hipótesis general}

Existen diferencias en el desempeño en los procesos psicológicos de la lectura en los estudiantes de la Provincia Constitucional del Callao según el nivel socioeconómico y el género.

\section{Hipótesis específicas}

- Existen diferencias en el desempeño en el proceso de identificación de letras (conocimiento del nombre o sonido de la letra, reconocer la palabra igualdiferente) en los estudiantes de la Provincia Constitucional del Callao según el nivel socioeconómico y el género.

- Existen diferencias en el desempeño en los procesos léxicos (dominio de la ruta fonológica y léxica) en los estudiantes de la Provincia Constitucional del Callao según el nivel socioeconómico y el género.

- Existen diferencias en el desempeño en los procesos sintácticos (conocimiento de la estructura gramatical y de los signos de puntuación) en los estudiantes de la Provincia Constitucional del Callao según el nivel socioeconómico y el género.

- Existen diferencias en el desempeño en los procesos semánticos de la lectura (comprensión de oraciones, comprensión de textos y comprensión oral) en los estudiantes de la Provincia Constitucional del Callao según el nivel socioeconómico y el género.

- Existen diferencias en el nivel de velocidad de la lectura los estudiantes de $1^{\circ}$ a $6^{\circ}$ grado de educación primaria de la Provincia Constitucional del Callao tomando en cuenta el nivel socioeconómico y el género. 


\section{MÉTODO}

El presente fue un estudio de tipo sustantivo descriptivo (Sánchez \& Reyes, 2006), en trataba de aportar a los problemas teoréticos o sustantivos sobre las medidas más adecuadas de evaluación de la lectura y por otro intentaba elaborar un perfil diagnóstico sobre el desempeño en la lectura que presentan los estudiantes de la provincia constitucional del Callao según el género y el nivel socioeconómico al cual pertenecen.

En cuanto al diseño se trató de una investigación descriptiva comparativa puesto que se dividió la realidad de estudio en cuatro niveles socioeconómicos tomando en cuenta el lugar y procedencia de las instituciones educativas del Callao y se evaluó el nivel de funcionamiento de cada uno de los procesos psicológicos de la lectura, estrategias y velocidad lectora.

\section{Participantes}

La población objetivo estuvo constituida por los niños y niñas de primero a sexto grado de primaria de la Provincia Constitucional del Callao que estudian tanto en las instituciones privadas y estatales. Para la muestra se consideró cuatro niveles socioeconómicos distintos tomando como criterio el tipo de escuela (pública o privada) y el lugar de procedencia de los alumnos (Millones, 2001; Piñeros \& Rodríguez, 1999, citado por Caro 2002). Estos fueron: medio-alto; medio-bajo; bajo y bajo-bajo según lo determinado por la Asociación Peruana de Empresas de Investigación de Mercados (APEIM, 2012).

Se utilizó el muestreo no probabilístico por cuotas polietápico, con selección de las unidades primarias de muestreo (nivel socioeconómico) por cuotas de forma proporcional, de las unidades secundarias (colegios) de forma intencional y de las unidades últimas (sujetos) por cuotas de sexo y grado. A partir de este procedimiento se seleccionaron 966 sujetos pertenecientes a los seis grados de educación primaria y a los cuatro niveles socioeconómicos de la población de tal forma que el $13 \%$ de la muestra debía pertenecer a un nivel socioeconómico medio (B), el $30 \%$ a un nivel socioeconómico medio bajo (C), el $31 \%$ a un nivel socioeconómico bajo (D) y el $26 \%$ a un nivel socioeconómico bajo bajo (E). Estos porcentajes se corresponden con los designados para cada nivel socioeconómico para la región del Callao según APEIM (2012). Se procedió luego a seleccionar los distritos más representativos de cada nivel socioeconómico, de tal manera, que se consideraron los distritos de: La Punta, Bellavista, La Perla, Callao, Carmen de La Legua y Ventanilla. Posteriormente, se seleccionaron los centros educativos de gestión privada y pública más representativos de cada distrito tomando en cuenta los resultados encontrados luego de la aplicación de la Ficha Socioeconómica aplicada a las instituciones educativas elaborada a partir de los criterios recomendados APEIM (op. cit).

El número final de estudiantes seleccionados se expresan de la siguiente manera: 
Procesos psicológicos de la lectura en estudiantes de primaria del Callao según nivel socioeconómico y género

Tabla 1. Distribución de los sujetos por nivel socioeconómico y grado educativo

\begin{tabular}{|c|c|c|c|c|c|c|c|c|c|}
\hline \multirow{2}{*}{\multicolumn{2}{|c|}{ NSE }} & \multicolumn{2}{|c|}{ B } & \multicolumn{2}{|c|}{$\mathrm{C}$} & \multicolumn{2}{|c|}{$\mathrm{D}$} & \multicolumn{2}{|c|}{$\mathrm{E}$} \\
\hline & & Varones & Mujeres & Varones & Mujeres & Varones & Mujeres & Varones & Mujeres \\
\hline \multirow{6}{*}{ Grado } & $1^{\circ}$ grado & 10 & 11 & 24 & 24 & 25 & 25 & 21 & 21 \\
\hline & $2^{\circ}$ grado & 10 & 11 & 23 & 25 & 23 & 27 & 21 & 21 \\
\hline & $3^{\circ}$ grado & 11 & 10 & 24 & 24 & 25 & 25 & 21 & 21 \\
\hline & $4^{\circ}$ grado & 10 & 11 & 26 & 22 & 25 & 25 & 21 & 21 \\
\hline & $5^{\circ}$ grado & 11 & 10 & 24 & 24 & 25 & 25 & 21 & 21 \\
\hline & $6^{\circ}$ grado & 10 & 11 & 24 & 24 & 28 & 22 & 21 & 21 \\
\hline & OTAL & \multicolumn{2}{|c|}{126} & \multicolumn{2}{|c|}{288} & \multicolumn{2}{|c|}{300} & \multicolumn{2}{|c|}{252} \\
\hline
\end{tabular}

$\mathrm{N}=966$

\section{Instrumentos de investigación}

Para evaluar el nivel de desempeño en cada uno de los procesos psicológicos de la lectura se utilizó la Prueba PROLEC-R de Cuetos, Rodríguez, Ruano y Arribas D. (2007) con adaptación y baremación realizada por los autores del presente estudio.

El PROLEC-R es una batería de aplicación individual para evaluar a niños de primero a sexto grado de educación primaria que consta de nueve tareas que exploran los principales procesos lectores (dos para cada uno de los procesos de la lectura, excepto el proceso semántico que consta con tres tareas). Estos son: Identificación de letras, procesos léxicos, procesos sintácticos y procesos semánticos. El PROLEC-R trata de averiguar qué componentes del sistema lector están fallando en los niños y niñas.

\section{RESULTADOS}

A continuación presentaremos los análisis inferenciales que responden a la siguiente hipótesis central y a las subhipótesis:

- Existen diferencias en desempeño en los procesos psicológicos de la lectura (identificación de letras, procesos léxicos, procesos sintácticos y procesos semánticos) en los estudiantes de la Provincia Constitucional del Callao según el nivel socioeconómico y el género.

- Existen diferencias en el nivel de velocidad de la lectura los estudiantes de $1^{\circ}$ a $6^{\circ}$ grado de educación primaria de la Provincia Constitucional del Callao tomando en cuenta el nivel socioeconómico y el género.

\section{Análisis inferencial}

Procesos Psicológicos de la Lectura y Nivel Socioeconómico 


\section{Identificación de letras}

El análisis inferencial se realizó a través del estadístico no paramétrico Kruskal Wallis que permite evidenciar si existen diferencias significativas entre los puntajes obtenidos en la identificación de letras en función del nivel socioeconómico. En la Tabla 2 se aprecian que existen diferencias significativas en el puntaje obtenido en la tarea de nombre de letras para todos los grados en cuanto al nivel socioeconómico, mientras que en la tarea igual-diferente solo se muestran diferencias significativas en el primer, segundo y tercer grado.

Tabla 2. Prueba de Kruskall Wallis para diferencias en la identificación de letras según el nivel socioeconómico.

\begin{tabular}{ccccc}
\hline \multirow{2}{*}{ Grado } & \multicolumn{2}{c}{ Nombre de letras } & \multicolumn{2}{c}{ Igual-diferente } \\
\cline { 2 - 5 } & Chi cuadrado & Sig & Chi cuadrado & Sig \\
\hline Primer grado & 91,84 &, 000 & 17,40 &, 001 \\
Segundo grado & 60,77 &, 000 & 11,73 &, 008 \\
Tercer grado & 88,00 &, 000 & 17,90 &, 000 \\
Cuarto grado & 27,08 &, 000 & 3,20 &, 361 \\
Quinto grado & 12,95 &, 005 & 3,78 &, 285 \\
Sexto grado & 17,75 &, 000 & 2,96 &, 398 \\
\hline
\end{tabular}

Asimismo, en la Tabla 3 se observan que existen diferencias significativas en el tiempo en segundos utilizado en la realización de las tareas de identificación de letras para todos los grados en cuanto al nivel socioeconómico, al ser el nivel crítico menor a 0,05 y por tanto, se infiere que la velocidad difiere en función del nivel socioeconómico.

Tabla 3. Prueba de Kruskall Wallis para diferencias en el tiempo en segundos utilizados en la identificación de letras según el nivel socioeconómico.

\begin{tabular}{ccccc}
\hline \multirow{2}{*}{ Grado } & \multicolumn{2}{c}{ Nombre de letras } & \multicolumn{2}{c}{ Igualdiferente } \\
\cline { 2 - 5 } & Chi cuadrado & Sig & Chi cuadrado & Sig \\
\hline Primer grado & 33,42 &, 000 & 20,04 &, 000 \\
Segundo grado & 39,30 &, 000 & 36,94 &, 000 \\
Tercer grado & 72,11 &, 000 & 13,33 &, 004 \\
Cuarto grado & 35,08 &, 000 & 47,24 &, 000 \\
Quinto grado & 38,65 &, 000 & 22,22 &, 000 \\
Sexto grado & 78,19 &, 000 & 71,43 &, 000 \\
\hline
\end{tabular}




\section{Procesos léxicos}

El análisis inferencial para la comparación de puntajes directos en el procesamiento léxico se muestra en la Tabla 4. En ella se puede evidenciar que existen diferencias significativas en el puntaje directo obtenido en la tarea de lectura de palabras en función al nivel socioeconómico para el primer, segundo y tercer grado de primaria, mientras que en la tarea lectura de pseudopalabras las diferencias son significativas para el primer, segundo, tercer y cuarto grado de primaria.

Tabla 4. Prueba de Kruskall Wallis para diferencias en los procesos léxicos según el nivel socioeconómico

\begin{tabular}{ccccc}
\hline \multirow{2}{*}{ Grado } & \multicolumn{2}{c}{ Lectura de palabras } & \multicolumn{2}{c}{ Lectura de pseudopalabras } \\
\cline { 2 - 5 } & Chi cuadrado & Sig & Chi cuadrado & Sig \\
\hline Primer grado & 48,99 &, 000 & 54,88 &, 000 \\
Segundo grado & 33,52 &, 000 & 28,59 &, 000 \\
Tercer grado & 43,64 &, 000 & 88,84 &, 000 \\
Cuarto grado & 7,52 &, 057 & 11,45 &, 009 \\
Quinto grado & 5,61 &, 132 & 5,18 &, 159 \\
Sexto grado & 5,24 &, 155 & 4,51 &, 211 \\
\hline
\end{tabular}

Asimismo, en la Tabla 5 se observan que existen diferencias significativas en el tiempo en segundos utilizado en la realización de la tarea de lectura de palabras para todos los grados en cuanto al nivel socioeconómico. Sin embargo, en la tarea de lectura de pseudopalabras solo existen diferencias significativas en el primer, segundo y tercer grado de primaria, ello estaría indicando que para los grados posteriores la velocidad en la lectura de pseudopalabras no difiere en función del nivel socioeconómico.

Tabla 5. Prueba de Kruskall Wallis para diferencias en el tiempo en segundos utilizados en los procesos léxicos según el nivel socioeconómico.

\begin{tabular}{ccccc}
\hline \multirow{2}{*}{ Grado } & \multicolumn{2}{c}{ Lectura de palabras } & \multicolumn{2}{c}{ Lectura de pseudopalabras } \\
\cline { 2 - 5 } & Chi cuadrado & Sig & Chi cuadrado & Sig \\
\hline Primer grado & 12,27 &, 007 & 9,16 &, 027 \\
Segundo grado & 28,59 &, 000 & 25,29 &, 000 \\
Tercer grado & 27,78 &, 000 & 19,89 &, 000 \\
Cuarto grado & 24,14 &, 000 & 3,04 &, 385 \\
Quinto grado & 22,80 &, 000 & 3,32 &, 344 \\
Sexto grado & 35,92 &, 000 & 14,86 &, 002 \\
\hline
\end{tabular}




\section{Procesos Sintácticos}

El análisis inferencial para la comparación de puntajes directos en el procesamiento sintáctico se muestra en la Tabla 6 . En ella se puede evidenciar que existen diferencias significativas en el puntaje directo obtenido en la tarea de signos de puntuación en función al nivel socioeconómico para todos los grados educativos, mientras que en la tarea de estructuras gramaticales las diferencias no son significativas para el sexto grado.

Tabla 6. Prueba de Kruskall Wallis para diferencias en los procesos sintácticos según el nivel socioeconómico

\begin{tabular}{ccccc}
\hline & \multicolumn{2}{c}{ Estructura Gramatical } & \multicolumn{2}{c}{ Signos de puntuación } \\
\cline { 2 - 5 } Grado & Chi cuadrado & Sig & Chi cuadrado & Sig \\
\hline Primer grado & 73,80 &, 000 & 110,21 &, 000 \\
Segundo grado & 35,31 &, 000 & 52,80 &, 000 \\
Tercer grado & 13,55 &, 000 & 74,36 &, 000 \\
Cuarto grado & 11,56 &, 009 & 36,03 &, 000 \\
Quinto grado & 12,20 &, 007 & 16,72 &, 001 \\
Sexto grado & 4,31 &, 230 & 28,28 &, 000 \\
\hline
\end{tabular}

De la misma forma, en la Tabla 7 se observan que existen diferencias significativas en el tiempo en segundos utilizado en la realización de la tarea de signos de puntuación para todos los grados, según el nivel socioeconómico.

Tabla 7. Prueba de Kruskall Wallis para diferencias en el tiempo en segundos utilizados en la tarea de signos de puntuación según el nivel socioeconómico.

\begin{tabular}{ccc}
\hline & \multicolumn{2}{c}{ Tiempo en segundos - Signos de puntuación } \\
\cline { 2 - 3 } Grado & Chi cuadrado & Sig \\
\hline Primer grado & 15,75 &, 001 \\
Segundo grado & 28,93 &, 000 \\
Tercer grado & 17,44 &, 001 \\
Cuarto grado & 31,68 &, 000 \\
Quinto grado & 12,93 &, 005 \\
Sexto grado & 32,38 &, 000 \\
\hline
\end{tabular}

\section{Procesos Semánticos}

El análisis inferencial para la comparación de puntajes directos en el procesamiento semántico se muestra en la Tabla 8. En ella se puede evidenciar que existen diferencias significativas en el puntaje directo obtenido en las tareas de comprensión de 
textos y comprensión oral en función al nivel socioeconómico para todos los grados educativos. En cuanto a la tarea de comprensión de oraciones existen diferencias significativas para todos los grados excepto para el quinto grado. Se puede concluir que el procesamiento semántico difiere en función del nivel socioeconómico.

Tabla 8. Prueba de Kruskall Wallis para diferencias en los procesos semánticos según el nivel socioeconómico

\begin{tabular}{ccccccc}
\hline & \multicolumn{2}{c}{ Comprensión Oraciones } & \multicolumn{2}{c}{ Comprensión Textos } & \multicolumn{2}{c}{ Comprensión Oral } \\
\cline { 2 - 7 } Grado & Chi cuadrado & Sig & Chi cuadrado & Sig & Chi cuadrado & Sig \\
\hline Primer grado & 78,10 &, 000 & 97,42 &, 000 & 28,42 &, 000 \\
Segundo grado & 23,65 &, 000 & 53,37 &, 000 & 28,46 &, 000 \\
Tercer grado & 20,96 &, 000 & 42,63 &, 000 & 53,85 &, 000 \\
Cuarto grado & 17,52 &, 001 & 46,47 &, 000 & 48,60 &, 000 \\
Quinto grado & 6,08 &, 107 & 29,95 &, 000 & 31,40 &, 000 \\
Sexto grado & 16,81 &, 001 & 29,96 &, 000 & 56,67 &, 000 \\
\hline
\end{tabular}

\section{Procesos psicológicos de la lectura y género}

\section{Identificación de letras}

El análisis inferencial para hallar las diferencias en los procesos psicológicos de la lectura en función del género se realizó a través del estadístico no paramétrico U de Mann Whitney. En la Tabla 9 se muestra el análisis comparativo de los puntajes obtenidos en las tareas de identificación de letras donde se aprecian que no existe diferencias significativas en el puntaje obtenido en la tarea de nombre de letras ni en la tarea igual-diferente en función del género

Tabla 9. Prueba de U de Mann Whitney para diferencias en la identificación de letras según el género

\begin{tabular}{cccccccc}
\hline \multicolumn{3}{c}{ Nombre de letras } & Igual-d \\
\hline $\begin{array}{c}\text { Rango } \\
\text { promedio } \\
\text { mujeres }\end{array}$ & $\begin{array}{c}\text { Rango } \\
\text { promedio } \\
\text { varones }\end{array}$ & U & Sig & $\begin{array}{c}\text { Rango } \\
\text { promedio } \\
\text { mujeres }\end{array}$ & $\begin{array}{c}\text { Rango } \\
\text { promedio } \\
\text { varones }\end{array}$ & U & Sig \\
\hline 473,81 & 491,31 & 120,3 &, 283 & 494,88 & 474,27 & 110,6 &, 194 \\
\hline
\end{tabular}

Asimismo, en la Tabla 10 se observa que no existe diferencias significativas en el tiempo en segundos utilizado en la realización de las tareas de identificación de letras, al ser el nivel crítico mayor a 0,05 y por tanto, la velocidad de la realización de las tareas de identificación de letras no difiere en función al género. 
Tabla 10. Prueba de U de Mann Whitney para diferencias en el tiempo en segundos utilizados en la identificación de letras según el género

\begin{tabular}{cccccccc}
\hline \multicolumn{3}{c}{ Nombre de letras } & Igual-diferente \\
\hline $\begin{array}{c}\text { Rango } \\
\text { promedio } \\
\text { mujeres }\end{array}$ & $\begin{array}{c}\text { Rango } \\
\text { promedio } \\
\text { varones }\end{array}$ & U & Sig & $\begin{array}{c}\text { Rango } \\
\text { promedio } \\
\text { mujeres }\end{array}$ & $\begin{array}{c}\text { Rango } \\
\text { promedio } \\
\text { varones }\end{array}$ & U & Sig \\
\hline 499,79 & 467,83 & 108,4 &, 075 & 482,15 & 467,49 & 108,6 &, 410 \\
\hline
\end{tabular}

\section{Procesos léxicos}

El análisis inferencial para la comparación de puntajes directos en el procesamiento léxico según el género se muestra en la Tabla 11. En ella se puede evidenciar que no existen diferencias significativas en el puntaje directo obtenido en la tarea de lectura de palabras, ni en la tarea de lectura de pseudopalabras en función al género.

Tabla 11. Prueba de U de Mann Whitney Wallis para diferencias en los procesos léxicos según el nivel género

\begin{tabular}{cccccccc}
\hline \multicolumn{3}{c}{ Lectura de palabras } & \multicolumn{5}{c}{ Lectura de pseudopalabras } \\
\hline $\begin{array}{c}\text { Rango } \\
\text { promedio } \\
\text { mujeres }\end{array}$ & $\begin{array}{c}\text { Rango } \\
\text { promedio } \\
\text { varones }\end{array}$ & U & Sig & $\begin{array}{c}\text { Rango } \\
\text { promedio } \\
\text { mujeres }\end{array}$ & $\begin{array}{c}\text { Rango } \\
\text { promedio } \\
\text { varones }\end{array}$ & U & Sig \\
\hline 483,53 & 482,53 & 115,8 &, 951 & 482,72 & 483,26 & 116,2 &, 975 \\
\hline
\end{tabular}

Asimismo, en la Tabla 12 se observa que tampoco existen diferencias significativas en el tiempo en segundos utilizado en la realización de las tareas de lectura de palabras y lectura de pseudopalabras en función al género, ello estaría indicando que la velocidad en la lectura no difiere en función del sexo.

Tabla 12. Prueba de U de Mann Whitney para diferencias en el tiempo en segundos utilizados en los procesos léxicos según el género.

\begin{tabular}{cccccccc}
\hline \multicolumn{3}{c}{ Lectura de palabras } & \multicolumn{5}{c}{ Lectura de pseudopalabras } \\
\hline $\begin{array}{c}\text { Rango prome- } \\
\text { dio mujeres }\end{array}$ & $\begin{array}{c}\text { Rango } \\
\text { promedio } \\
\text { varones }\end{array}$ & U & Sig & $\begin{array}{c}\text { Rango } \\
\text { promedio } \\
\text { mujeres }\end{array}$ & $\begin{array}{c}\text { Rango } \\
\text { promedio } \\
\text { varones }\end{array}$ & U & Sig \\
\hline 482,49 & 451,89 & 101,2 &, 083 & 469,09 & 456,48 & 103,5 &, 473 \\
\hline
\end{tabular}

\section{Procesos sintácticos}

El análisis inferencial para la comparación de puntajes directos en el procesamiento sintáctico en función del género se muestra en la Tabla 13. En ella se puede 
evidenciar que no existen diferencias significativas en el puntaje directo obtenido en la tarea de estructuras gramaticales, ni en la tarea de signos de puntuación tomando en cuenta el género.

Tabla 13. Prueba de U de Mann Whitney para diferencias en los procesos sintácticos según el género

\begin{tabular}{cccccccc}
\hline \multicolumn{3}{c}{ Estructuras gramaticales } & \multicolumn{5}{c}{ Signos de puntación } \\
\hline $\begin{array}{c}\text { Rango prome- } \\
\text { dio mujeres }\end{array}$ & $\begin{array}{c}\text { Rango } \\
\text { promedio } \\
\text { varones }\end{array}$ & U & Sig & $\begin{array}{c}\text { Rango } \\
\text { promedio } \\
\text { mujeres }\end{array}$ & $\begin{array}{c}\text { Rango } \\
\text { promedio } \\
\text { varones }\end{array}$ & U & Sig \\
\hline 465,82 & 483,35 & 116,4 &, 322 & 484,72 & 480,49 & 114,8 &, 607 \\
\hline
\end{tabular}

De la misma forma, en la Tabla 14 se observa que tampoco existen diferencias significativas en el tiempo en segundos utilizado en la realización de las tareas de estructuras gramaticales y signos de puntuación según el género.

Tabla 14. Prueba de U de Mann Whitney para diferencias en el tiempo en segundos utilizados en la tarea de signos de puntuación según el género

\begin{tabular}{cccc}
\hline \multicolumn{4}{c}{ Signos de puntuación } \\
\hline Rango promedio mujeres & Rango promedio varones & $\mathrm{U}$ & Sig \\
\hline 460,09 & 448,44 & 99,98 &, 504 \\
\hline
\end{tabular}

\section{Procesos semánticos}

El análisis inferencial para la comparación de puntajes directos en el procesamiento semántico en función del género se muestra en la Tabla 15. En ella se puede evidenciar que no existen diferencias significativas en el puntaje directo obtenido en las tareas de comprensión de oraciones y comprensión de textos, mientras que sí existen diferencias en la tarea de comprensión oral en función al sexo, a favor de los varones.

Tabla 15. Prueba de U de Mann Whitney para diferencias en los procesos semánticos según el género

\begin{tabular}{|c|c|c|c|c|c|c|c|c|c|}
\hline & \multicolumn{3}{|c|}{ Comprensión de oraciones } & \multicolumn{3}{|c|}{ Comprensión de textos } & \multicolumn{3}{|c|}{ Comprensión oral } \\
\hline & $\begin{array}{l}\text { Rango } \\
\text { prome- } \\
\text { dio }\end{array}$ & $\mathrm{U}$ & Sig. & $\begin{array}{l}\text { Rango } \\
\text { prome- } \\
\text { dio }\end{array}$ & $\mathrm{U}$ & Sig. & $\begin{array}{l}\text { Rango } \\
\text { prome- } \\
\text { dio }\end{array}$ & U & Sig. \\
\hline Mujeres & 476,60 & 117,20 & ,621 & 471,60 & 119,39 &, 318 & 448,10 & 130,08 &, 000 \\
\hline Varones & 485,01 & & & 489,45 & & & 510,59 & & \\
\hline
\end{tabular}




\section{DISCUSIÓN}

En el primer proceso, identificación de letras, examinado a través de dos tareas: decir el nombre o sonido de las letras y reconocer si un par de palabras son iguales o diferentes, se encontraron diferencias significativas según nivel socioeconómico en las tareas de nombre o sonido de letras en todos los grados y en igual - diferente en el primer, segundo y tercer grado. Relacionando este resultado con lo que ocurre con la velocidad lectora, donde también aparecen claras diferencias a favor del sector socio- económico más favorecido, queda claro que los estudiantes de los sectores más pobres demoran más en decir el nombre o los sonidos de las letras por falta de automaticidad en dicha tarea, lo cual, desde nuestro punto de vista, tiene que ver directamente con un problema de método en la enseñanzaaprendizaje de la lectura, que afecta principalmente a los niños de las instituciones educativas estatales.

Al respecto, es bueno mencionar, que Urquijo (2009) realizó un estudio similar en Argentina con 610 estudiantes utilizando la Batería de Evaluación de los Procesos Lectores (Cuetos, Rodríguez, Ruano, 1996). En el caso del proceso de identificación de letras no encontró diferencias significativas según el nivel socioeconómico ni siquiera en los primeros grados. Este hallazgo no se corresponde con lo encontrado en nuestro estudio. Una de las razones sería que en Argentina, tanto en las instituciones educativas públicas y privadas, se utiliza una metodología común para la enseñanza de la lectoescritura. Sin embargo, en nuestro país, en las instituciones educativas particulares los docentes de aula no necesariamente aplican las orientaciones metodológicas del diseño curricular nacional del Ministerio.

En cuanto a los procesos léxicos evaluados a través de dos tareas: lectura de palabras y lectura de pseudopalabras, se encontraron diferencias significativas según el nivel socioeconómico en la tarea de lectura de palabras en el primer, segundo y tercer grado de primaria y en la tarea de lectura de pseudopalabras en el primer, segundo, tercer y cuarto grado de primaria. Este resultado podemos relacionarlo también con las diferencias significativas halladas, tomando en cuenta la velocidad invertida en la lectura usando la ruta visual-ortográfica. Mientras que en el caso de las pseudopalabras (palabras sin significado que solo pueden decodificarse por la ruta fonológica) las diferencias en la velocidad se presentaron en primero, segundo, tercer y sexto grado de primaria. Es decir, no se presentan diferencias en la velocidad en el cuarto y quinto grado en el uso de la ruta fonológica. Si comparamos el promedio de velocidad en la lectura de palabras con la de pseudopalabras observamos que las diferencias (que debería ser notorias mientras más se avanza en el grado escolar) no se presentan tan marcadas en los niveles socioeconómicos más deprimidos mientras que en los sectores más favorecidos la velocidad invertida en la lectura de palabras aventaja a la de pseudopalabras. Esto significa que estos estudiantes han construido un diccionario léxico mental que les permite que las palabras conocidas sean leídas al instante. En este proceso tampoco se encontraron diferencias según el género, es decir que la única variable que marca las diferencias en el perfil de decodificación lectora es la socioeconómica. Estos hallazgos se corroboran con el estudio de Salas \& Villanueva (2012) quienes también observaron 
diferencias significativas en la lectura de palabras y pseudopalabras comparando dos instituciones educativas con distinto tipo de gestión.

En cuanto a los procesos sintácticos examinados a través de dos tareas: estructuras gramaticales y signos de puntuación, al encontrar en la primera, diferencias significativas en todos los niveles socioeconómicos y en todos los grados, menos en el sexto grado, pensamos que ello podría estar relacionada con la falta de estimulación del lenguaje oral que en las etapas tempranas sufren los niños más desfavorecidos. Como se sabe, el desarrollo del lenguaje oral depende de la calidad y cantidad de interacciones lingüísticas madre-niño (González, 1995a) a partir de las cuales se van construyendo los subsistemas del lenguaje, siendo uno de ellos, el sintáctico. En la segunda tarea ,signos de puntuación, también se encontraron diferencias significativas en todos los grados en función al nivel socioeconómico. Estas diferencias se mantuvieron cuando se tomó en cuenta la velocidad invertida en dicha tarea siendo los sujetos de sectores más desfavorecidos, aquellos que invirtieron más tiempo en esta demanda. En cuanto al género no se encontraron diferencias significativas en la tarea de estructuras gramaticales, ni en signos de puntuación, ni en la velocidad que esta tarea demanda. El hallazgo sobre diferencias por nivel socio- económico, se relaciona con lo encontrado por Céspedes \& Morales (2011) quienes también hallaron diferencias según nivel socioeconómico en niños y niñas de 8 y 9 años de edad que estudiaban en tres instituciones educativas ubicadas en la UGEL 06 de la ciudad de Lima.

En cuanto al proceso semántico, evaluado a través de tres tareas: comprensión de oraciones, comprensión de textos y comprensión oral, se hallaron diferencias significativas según nivel socioeconómico en las tareas de comprensión de textos y comprensión oral en todos los grados. En la tarea de comprensión de oraciones también se encontraron diferencias significativas en todos los grados excepto en el quinto grado. Solo en quinto grado no se hallaron diferencias significativas según el nivel socioeconómico mientras que en el resto de grados se observaron promedios distintos. Es decir, que aún en la tarea menos exigente se observaron diferencias según el nivel socioeconómico. La comprensión de textos se evaluó con preguntas inferenciales que el examinado debe contestar luego de leer cuatro textos: dos narrativos y dos expositivos. Todas las preguntas que se efectúan son de tipo inferencial. Se halló diferencias significativas en las cuatro realidades socioeconómicas. Esto corrobora los resultados de las evaluaciones internacionales y nacionales en comprensión lectora donde los estudiantes peruanos de sectores desfavorecidos alcanzan bajos niveles de rendimiento frente a los más aventajados. Por último, tenemos a la comprensión oral. En este estudio se observan bajos niveles de rendimiento en la comprensión oral en los cuatro niveles socioeconómicos seleccionados. Es decir, se evidencia la reducida estimulación de esta competencia en todos los grados de primaria en nuestras instituciones educativas. Es importante resaltar que solo se encontraron diferencias significativas según género en comprensión oral a favor de los varones. Esto contraviene todas las investigaciones tradicionales donde siempre fueron las niñas que obtenían mayores puntajes que los varones en las tareas del lenguaje oral. Al parecer la dinámica actual impuesta 
por las sociedades post modernas podría explicar dicho fenómeno. La exigencia laboral, la desmotivación y el reducido apego estarían relacionados con una menor estimulación lingüista por parte de la madre hacia la hija. La ventaja de los varones frente a las mujeres también fue encontrada en el estudio piloto realizado por los autores de la prueba (2007) quienes hallaron diferencias significativas a favor de los varones en comprensión oral, comprensión de oraciones y comprensión de textos a un nivel de confianza del $99 \%$.

\section{CONCLUSIONES}

\section{Diferencias según el nivel socioeconómico}

1. En cuanto al proceso de identificación de letras se encontró diferencias significativas según nivel socioeconómico en las tareas de nombre de letras en todos los grados y en igual-diferente en el primer, segundo y tercer grado.

2. Considerando los procesos léxicos se encontraron diferencias significativas según nivel socioeconómico en la tarea de lectura de palabras entre el primero a tercer grado de primaria y en la tarea de lectura de pseudopalabras en el primero hasta el cuarto grado de primaria. Esto revelaría el bajo dominio inicial que presentan los estudiantes más desfavorecidos en el uso la ruta fonológica y visual-ortográfica de la lectura.

3. Con respecto a los procesos sintácticos se encontraron diferencias significativas según nivel socioeconómico en la tarea de signos de puntuación en todos los grados y en la tarea de estructuras gramaticales menos en sexto.

4. En cuanto al proceso semántico se hallaron diferencias significativas según nivel socioeconómico en las tareas de comprensión de textos y comprensión oral en todos los grados y en la comprensión de oraciones también, menos en quinto.

\section{Diferencias según velocidad lectora}

5. En cuanto a la velocidad lectora en los procesos de identificación de letras, se encontró diferencias significativas en sus dos tareas: nombre de letras e igual-diferente en todos los grados y en los cuatro niveles socioeconómicos. Estas diferencias en la velocidad no se encontraron cuando se compararon los promedios tomando en cuenta el género de los estudiantes.

6. En los procesos léxicos se encontraron diferencias significativas en el tiempo en segundos utilizado en lectura de palabras en todos los grados y en la tarea de lectura de pseudopalabras en el primer, segundo, tercer y sexto grado de primaria.

7. En los procesos sintácticos en la tarea de signos de puntuación se encontraron diferencias en el tiempo utilizado en todos los grados y niveles socioeconómicos, lo que revelaría una falta de dominio de este subproceso de alto nivel en los sectores menos aventajados. 
Procesos psicológicos de la lectura en estudiantes de primaRia del Callao según nivel SOCIOeConÓmico y género

\section{Diferencias según género}

8. No se encontraron diferencias significativas en el proceso de identificación de letras en las tareas de nombre de letras e igual-diferente según género.

9. En cuanto a los procesos léxicos tampoco se encontraron diferencias significativas en la tarea de lectura de palabras, ni de pseudopalabras en función al género.

10. En lo concerniente al procesamiento sintáctico no se encontraron diferencias significativas en la tarea de estructuras gramaticales, ni en la tarea de signos de puntuación tomando en cuenta el género.

11. Por último, en el procesamiento semántico tampoco se encontraron diferencias significativas en las tareas de comprensión de oraciones y comprensión de textos, pero sí se encontraron diferencias significativas en la tarea de comprensión oral según género a favor de los varones, lo cual contradice estudios anteriores donde las niñas aventajaban siempre a los varones quienes, al parecer, tienen ahora más oportunidades que antes de interactuar lingüísticamente en su medio.

\section{Nota de reconocimiento}

Queremos reconocer y agradecer a los estudiantes de Psicología tanto de la Universidad Nacional Mayor de San Marcos como de la Universidad Federico Villarreal que colaboraron eficientemente para hacer posible este trabajo de investigación: Edson Huerta R., Lizet Fernández C., Alex Casariego L., Sandra Meléndez J. y Yamely Cárdenas M.

También, a los directores, profesores y estudiantes de los colegios del Callao que nos abrieron las puertas de sus instituciones educativas.

\section{REFERENCIAS BIBLIOGRÁFICAS}

APEIM: Asociación Peruana de Empresas de Investigación de Mercados (2012).

Niveles socioeconómicos 2012. Total Perú urbano y Lima Metropolitana. Lima. http:// www.apeim.com.pe/images/APEIM_NSE_2012.pdf.

Bravo, L. (2005b). Lenguaje escrito y dislexias. Enfoque cognitivo del retardo lector. Cuarta edición ampliada. Santiago de Chile. Ediciones Universidad Católica de Chile.

Caro, D. (2002). Estimación del nivel socioeconómico de las familias: Propuesta metodológica para la Evaluación Nacional de Rendimiento del 2011. Recuperado de http:// www2.minedu.gob.pe/umc/admin/images/.../menanexos_44.pdf

Canales, R. (2007). Comprensión Lectora y problemas de aprendizaje: un enfoque cognitivo. Perú: CONCYTEC.

Céspedes B. \& Morales, A. (2011). Diferencias en la comprensión lectora según la edad, género y nivel socioeconómico en tres instituciones educativas de Lima. Tesis para optar el grado de Magíster. Pontificia Universidad Católica del Perú. Lima, Perú. 
Cuetos, F. (2008). Psicología de la lectura. Educación infantil y primaria. España: Ediciones Wolters Kluwer.

Cuetos F., Rodríguez B., Ruano E., \& Arribas D. (2007). PROLEC-R: Batería de evaluación de los procesos lectores, revisada. Madrid: TEA.

Cuetos, F. \& Ramos S. (1999). Evaluación de los procesos lectores en alumnos del tercer ciclo de Educación Primaria y secundaria PROLEC-SE. Madrid: TEA.

Cuetos, F., Rodríguez, B. \& Ruano, E. (1996). PROLEC: Evaluación de los procesos lectores. Madrid: TEA.

Dioses Ch. A., Evangelista Z.; C., Basurto T.A., Morales C. M., Alcántara, P.M. (2010). Procesos cognitivos implicados en la lectura y escritura de niños y niñas del tercer grado de educación primaria residentes en Lima y Piura. En Revista del Instituto de Investigaciones Psicológicas UNMSM. Volumen 13. N. ${ }^{\circ} 1$, pp. 13-40.

García V. \& González M. (2000). Dificultades de Aprendizaje e Intervención Psicopedagógica (Vol. 2.). Lectura y Escritura. Madrid, España. Editorial EOS.

González M., R. (1993). Bilingüismo y problemas de aprendizaje. Revista de Problemas de aprendizaje. C.E.P. Palestra; Año 3, N. ${ }^{\circ} 1$.

González M., R. (1995a). Exploración del desarrollo del lenguaje en el niño peruano menor de 3 años: Un modelo interactivo. Proyecto de Innovaciones Pedagógicas No-Formales. Fundación Bernad Van Leer, Ministerio de Educación.

González M., R. (1995c). Psicología del Niño Peruano. Universidad de Lima. Facultad de Psicología.

Ministerio de Educación del Perú (2009). Diseño Curricular Nacional de Educación Básica Regular.

Ministerio de Educación del Perú. (2011). Evaluación Censal de Estudiantes 2011. Informe de Resultados para el Docente. Recuperado de: http://www2.minedu.gob.pe/umc

OCDE. Organización para la Cooperación y el Desarrollo Económico (2009). El programa PISA de la OCDE. Qué es y para qué sirve. En: www.oecd.org/dataoecd/58/51/39730818.pdf

Sánchez H. y Reyes C. (2006). Metodología y diseños en la investigación científica. Editorial Universitaria. Universidad Ricardo Palma.

Urquijo, S. (2009). El desarrollo de las funciones ejecutivas, la atención y el aprendizaje de la lectura. Facultad de Psicología, Universidad Nacional de Mar de Plata.

Velarde E. (2001). Relación entre la conciencia fonológica y el nivel de Decodificación y comprensión lectora en niños de 8 años del 3 er $^{\text {er }}$ grado de primaria de dos niveles socio-económicos del Cercado del Callao. Tesis para obtener el grado de Magíster en Educación. UNIFÉ, Lima Perú.

Velarde, E. \& Canales, R. (2008). La lectura en el Perú: Drama y Esperanza. Lima: Ediciones. Universidad Enrique Guzmán y Valle, La Cantuta.

Velarde, E., Canales, R., Meléndez C. y Lingán S. (2009). Diseño y ejecución de un programa de intervención para el mejoramiento de la lectura escritura en niños de la 
Procesos psicológicos de la lectura en estudiantes de primaria del Callao según nivel socioeconómico y género

Provincia del Callao. Ponencia presentada a X Congreso Latinoamericano de Lectura de Lectura y Escritura.

Wimmer, H. (1993). Characteristics of developmental dyslexia in a regular writing system. Applied Psycholinguistics, 14, 1-33. 